\title{
Reading from Scratch - A Vision-System for Reading Data on Micro-structured Surfaces
}

\author{
Ralf Dragon, Christian Becker, Bodo Rosenhahn, and Jörn Ostermann \\ Institut für Informationsverarbeitung \\ Leibniz Universität Hannover \\ Appelstraße 9a, 30167 Hannover, Germany \\ \{dragon, becker, rosenhahn, ostermann\}@tnt. uni-hannover.de
}

\begin{abstract}
Labeling and marking industrial manufactured objects gets increasingly important nowadays because of novel material properties and plagiarism. As part of the Collaborative Research Center 653 which investigates micro-structured metallic surfaces for inherent mechanical data storage, we research into a stable and reliable optical readout of the written data. Since this comprises a qualitative surface reconstruction, we use directed illumination to make the micro structures visible. Then we apply a spectral analysis to obtain image partitioning and perform signal tracking enhanced by a customized Hidden Markov Model. In this paper, we derive the algorithms used and demonstrate reading data from a surface with $1.6 \mathrm{kbit} / \mathrm{cm}^{2}$ from a micro-structured groove which varies by only $3 \mu \mathrm{m}$ in depth (thus a "scratch"). We demonstrate the system's robustness with experiments with real and artificially-rendered surfaces.
\end{abstract}

\section{Introduction}

In this paper an optical shape reconstruction method for the readout of data mechanically written as micro-structure on a surface is presented. The purpose is to store information about a mechanical component on the component itself. Since component and information then form a unit, the information cannot get lost or is unnecessarily stored even if the component has been replaced. Such

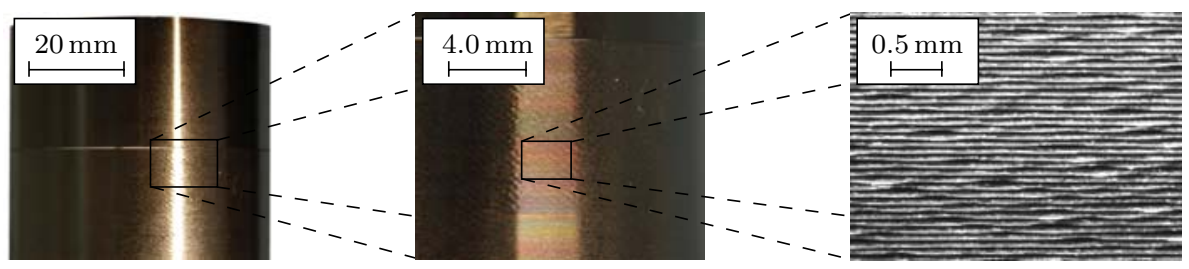

Fig. 1. Mechanical component with micro-structured surface

\footnotetext{
* This work was funded as part of the Sonderforschungsbereich 653 by the DFG.
} 

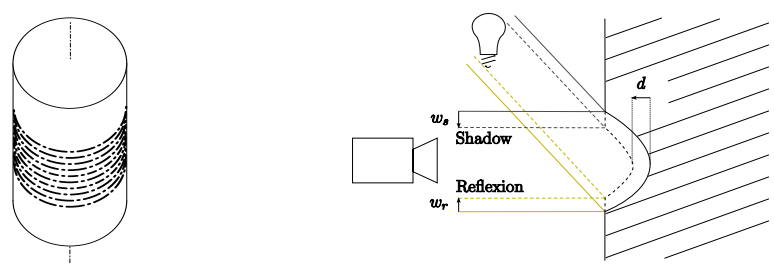

Fig. 2. Left: Groove wound around the micro-structured mechanical component. Right: Measuring principle of directed illumination: Varying depth $d$ is detected by shifts $w_{r}$ and $w_{s}$ of the reflexion and the shadow border respectively.

micro-structured surfaces are created by a Piezo tool during the last step of a turning process 1 . The tool cuts in a groove wound around the component whose depth is modulated by the digital payload. As the cutting is performed with small mechanical feed, the groove forms a micro-structure spreading on the whole surface of the mechanical component (Fig. 1). The mechanical storage principle used is the same as for the phonograph cylinder, the gramophone record or the capacitive electronic disc [2]. Unlike optical data storage systems like e.g., bar code, DVD or 3D storage [3], the domain of the applied signal is analog. To enable digital data storage, the run of the groove as well as writing and reading is modeled as the channel of a communication system [4, which consists of coding, modulation and channel. So writing in a groove means freezing an analog signal run as an imprint which is to be recovered during the shape reconstruction.

Since the micro-structures are designed for simple and fast writing during the production and insensitivity to mechanical stress, data density is low compared to state-of-the-art magnetic and optical storage media but sufficient for all labeling purposes. As rough estimate about the dimensions of a cutting parameter set suitable for writing and reading: mechanical feed $d_{f}=70 \mu \mathrm{m}$, groove depth $=$ $6 \mu \mathrm{m}$, signal amplitude $=3 \mu \mathrm{m}$, data rate $R=1.1 \mathrm{bit} / \mathrm{mm}$. When using a binary amplitude modulation ( 2 -ASK), the data density is $R \times 1 / d_{f} \approx 1.6 \mathrm{kbit} / \mathrm{cm}^{2}$.

\subsection{Previous Works}

Reading the information comprises at least a qualitative surface analysis to reconstruct the signal written in. The reconstruction should be possible in a cheap way so a mechanical component could be inspected at a service station. Thus, only shape analysis methods with a customary stereo microscope with low magnification are considered here which later can be adapted to hand-held units.

In the past 30 years, there were many shape reconstruction methods developed. In the field of microscopy with a small field of view, shape reconstruction is eased as only a depth map needs to be created. Thus, in the following we use the term depth instead of shape. Two main measuring principles are used: depth of field and perspective. It can be further distinguished between single-view and multi-view methods.

Depth of field as measuring principle utilizes that the image varies in sharpness, depending on the distance between camera and surface. Thus, the small 
depth of field of microscopes means high depth resolution. Depth from focus [5] is a multi-view method which builds a depth map by varying the object's distance and assigning all visible regions in focus to the same depth slice. As the distance variation is calibrated, points from different depth slices can be combined to a depth map. Depth from defocus [6] is a single-view method which makes use of the knowledge about the point spread function of the optical system to deduce depth from unsharpness.

Perspective as measuring principle means either using perspective differences in different surface views or analyzing illumination effects. Both types use knowledge about the set-up geometry. Depth from stereo [7] builds a depth map of the surface using a stereo microscope observing the same image from different perspectives. Using the pixel-wise found disparity along the epipolar line and the knowledge about the relation of both cameras, the depth map can be reconstructed. Depth from shading [8] makes use of the reflectivity function which relates knowledge about the set-up with the direction of the surface normal. Depth from directed illumination is similar to that. The surface is illuminated in such a way that only regions with a specific surface normal appear bright. The shadow and reflexion borders are used to deduce depth information.

\subsection{Surface Reconstruction at Scratch Scale}

During our research, we investigated three promising methods for surface reconstruction: depth from stereo (DFS), depth from focus (DFF) and depth from directed illumination (DFI). To the best of our knowledge, similar applications of depth reconstruction are usually one magnitude larger in size. E.g., the optical reconstruction of LP records 9] using DFF seems similar. However the depth of the groove is about 20 times and the groove distance about 24 times bigger than here. Another example is the optical inspection of solder joints [10] using depth from shading, where the solder joint height is about 25 times greater than the structures which are to be recovered here. Third example: The surface structures analyzed by [1] using DFF are 10 times deeper than our groove.

It turned out that DFS as well as DFF were able to reconstruct the coarse cylindrical shape of the mechanical component. However, both methods failed to reconstruct the groove which is about 10 times smaller. For DFS, one major problem is the calibration of the focal length with a microscopic calibration pattern, which is very important for exact depth estimation. It is error-prone as nearly no perspective effects are noticeable. DFF is heavily perturbed by mechanical inaccuracies when changing the focus depth. Another problem for both approaches is the highly-specular surface with many perturbations.

The measuring principle used for DFI here is displayed in Fig. 2 A depth variation of the groove is deduced by a shift of the reflexion and the shadow border. As this effect is even noticeable on low-magnification views, e.g., right image of Fig. 1, where DFS and DFF failed, we decided for this method. Under the assumption that the groove depth is proportional to the shift of the shadow border, the groove depth can be reconstructed qualitatively. The two key problems which are to be solved for this are the image partitioning, which is necessary 
to determine the position of the groove, and the tracking of the reflexion border for a robust depth reconstruction.

In this paper, we present the following contributions using DFI: In Section 2 the method to determine the position of the groove is explained. In Section 3 , the method to qualitatively reconstruct the analog signal using a Hidden Markov Model with a regular topology is presented. Both methods are then evaluated in Section 4 and the readout of a real surface is demonstrated. In Section 5, a conclusion is given.

\section{Image Partitioning Using Spectral Analysis}

We start with the first key problem of the groove reconstruction: Given one view of the surface with proper illumination like in Fig. 3, we want to extract the position of the groove containing the data. Given that the groove is wound around the mechanical component, several groove sections are visible. Each contains a different part of the signal run. As the mechanical feed during the turning process is constant, all neighbored groove sections have distance $\lambda$. We assume they run approximately horizontal in the microscope view, forming periodic coarse structures in vertical direction. The vertical position

$$
y_{n}=n \lambda-\phi
$$

of the $n$th groove center can be determined by analyzing the image texture in vertical direction to extract the groove distance $\lambda$ and the phase $\phi$.

The $1 \mathrm{D}$ texture analysis is based on several vertical cuts through the image. In order to just analyze the coarse structure and thus remove the impact of the fine structure, which contains the signal, we average these cuts to form the one-dimensional signal $f(y)$. The idea of the texture analysis is to model $f(y)$ as a cosine function and to find the sinusoidal parameters using a maximum likelihood (ML) estimation of the power spectrum density (PSD).

The PSD $p(u)$ of $f(y)$ is estimated using the average periodogram method [12, p 72ff.]. This means averaging PSD estimates of $r$ different realizations using a windowed discrete Fourier transform (DFT). Here, parts of $f(y)$ with length $s_{w}$ around varying positions $\xi$ are analyzed. Each PSD estimate $\mid\left(g_{\xi}(u) \mid\right.$ is:

$$
g_{\xi}(u)=\sum_{k=0}^{s_{w}-1} w(k) f\left(k+\xi-\frac{s_{w}-1}{2}\right) W_{s_{w}}^{-u}, \text { with } W_{s_{w}}=e^{j \frac{2 \pi}{s_{w}}} .
$$

We choose a Blackman-Harris Window [13] as windowing function $w(k)$ of odd size $s_{w}$. Since PSD estimates are shift-invariant, $p(u)$ and the ML estimate are:

$$
p(u)=\frac{1}{r} \sum_{i=0}^{r-1}\left|g_{\xi_{r}}(u)\right|, \quad(3) \quad u_{\mathrm{ml}}=\arg _{u} \max p(u), \quad(4) \quad \lambda=\frac{s_{w}}{u_{\mathrm{ml}}} .
$$

As the following phase computation requires a very exact estimation of $\lambda$, the ML estimation is followed by a maximum search with bounds $u_{\mathrm{ml}} \pm 1$. These 
are the preceding and following DFT sampling positions which have lower magnitudes.

Now the wavelength of our sinusoidal parameter estimation is known and the phase $\phi$ is to be determined. It is obviously not shift-invariant and thus may not be averaged like in (3); so PSD estimation does not include phase estimation. When using sinusoidal estimation to determine the phase, usually only a single realization is used which in our case is too much influenced by noise. In the following step, we combine the idea of averaging multiple realizations with the ML sinusoidal parameter estimation.

As $g_{\xi}(u)$ is found by taking different parts of $f(y)$, the phase estimate

$$
\phi_{\xi}=\frac{s_{w}}{2 \pi} \angle g_{\xi}\left(u_{\mathrm{ml}}\right)
$$

of different realizations can be combined and averaged later when compensating the shift of $\xi$ using

$$
\phi_{\xi}^{\prime}=\left(\phi_{\xi}-\xi+\frac{s_{w}-1}{2}\right) \bmod \lambda, \quad \text { (7) } \quad \phi=\frac{1}{r} \sum_{i=0}^{r-1} \phi_{\xi_{r}}^{\prime} .
$$

The interval $[0 \ldots \lambda]$ used in (7) could be detrimental for the averaging in the case of phase jumps. A more stable estimate is found when setting the interval borders symmetrically around median $\phi^{\prime}$, setting the lower border to $\alpha=$ median $\phi^{\prime}-\lambda / 2$ and recompute using

$$
\phi_{\xi}^{\prime \prime}=\left(\phi_{\xi}-\xi+\frac{s_{w}-1}{2}+\alpha\right) \bmod \lambda-\alpha, \quad(9) \quad \phi=\frac{1}{r} \sum_{i=0}^{r-1} \phi_{\xi_{r}}^{\prime \prime} .
$$

As distance and phase are known, the groove positions can be computed using (1). In reality, the groove centers do not run exactly horizontal as the mechanical component wobbles when being turned (visible in Fig. 8). Thus, one estimate is not enough for a whole surface image. We start from an initial estimate $\left(\lambda_{0}, \phi_{0}\right)$ at position $x_{0}$ and then iteratively connect estimate $k+1$ with $k$ using interval borders $\phi_{k} \pm \lambda_{k} / 2$ for correct phase unwrapping of $\phi_{k+1}$. In Fig. 3. an image partitioning using one estimate at the left and one at the right image border is displayed.

\section{Signal Tracking Using a Hidden Markov Model}

After the image partitioning, image parts of size $s_{x} \times s_{y}$ (where $s_{y}=\lceil\lambda\rceil$ ) containing exactly one groove section are created. The direction of the illumination has been chosen such that the lower wall of the groove wall appears bright (see Fig. 3). The task to solve the second key problem is tracking the reflexion border from the left to the right side of the image part. We will now deduce the Hidden Markov Model (HMM) used for the signal tracking.

A heuristic approximation for the observation probability of the groove's edge in image column $x_{i}$ being at position $y_{i}$ is the negative discrete deviation of the image $I$ in vertical direction, shifted by a constant $c$ to be non-negative: 
Fig. 3. Partitioned image with groove middle (dotted green) and tracked signal (blue)

$$
p_{o}\left(x_{i}, y_{i} \mid I\right) \propto-\frac{I\left(x_{i}, y_{i}+1\right)-I\left(x_{i}, y_{i}-1\right)}{2}+c .
$$

The transition probability of the actor's movement is modeled as a normal distribution with a maximum probability if the groove is constantly continued:

$$
p_{t}\left(x_{i+1}, y_{i+1} \mid y_{i}\right) \propto \mathcal{N}\left(\sigma^{2}, y_{i}\right) .
$$

Both $p_{o}$ as well as $p_{t}$ have to be normalized over one column as there is always exactly one state per column passed when traversing from the left to the right image side.

$$
\begin{array}{ll}
\sum_{j=1}^{s_{y}} p_{o}\left(x_{i}, y_{i}=j \mid I\right)=1, & \forall i=1 \ldots s_{x} \\
\sum_{j=1}^{s_{y}} p_{t}\left(x_{i+1}, y_{i+1}=j \mid y_{i}\right)=1, & \forall i=1 \ldots s_{x}-1, y_{i}=1 \ldots s_{y}
\end{array}
$$

Given $p_{o}$ and $p_{t}$, the HMM is established. The state $S_{x, y}$ stands for the groove's edge being at position $(x, y)$, so there are $s_{x} \times s_{y}$ states. The model's topology is adapted to the task: All states $S_{x_{i}, y_{i}}$ in the same image column $x_{i}$ are parallel; transitions are only possible to $S_{x_{i}+1, y_{i+1}}$ (Fig. 44).

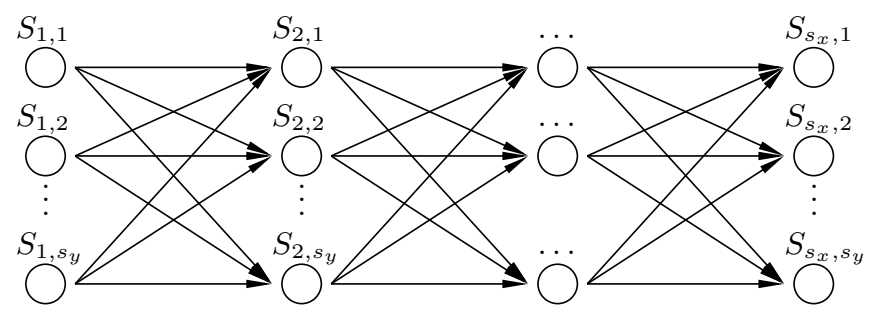

Fig. 4. The model's topology. Each state $S_{x, y}$ corresponds to the image pixel $I(x, y)$

Under these premises, the most probable groove edge $Y=\left(y_{1}, y_{2}, \ldots, y_{s_{x}}\right)$ is found by maximizing the overall transition probability

$$
P=p_{o}\left(x_{s_{x}}, y_{s_{x}}\right) \prod_{i=1}^{s_{x}-1} p_{t}\left(x_{i+1}, y_{i+1} \mid y_{i}\right) p_{o}\left(x_{i}, y_{i}\right)
$$



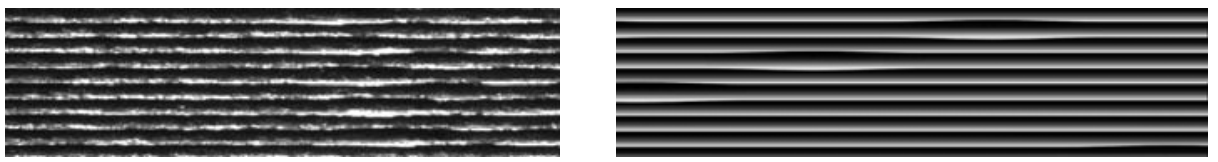

Fig. 5. Left: Original surface view with deepenings on the right half. Right: Povray rendering with deepenings in the top-left quarter.

Because of the simple and regular topology, $Y$ is easy to compute as a Viterbi algorithm [14] has always exactly $s_{y}$ paths to follow at the same time. The only parameter that has to be known a priori is the state transition standard deviation $\sigma$ which depends on the cutting parameters and the surface quality. As its influence is relatively small for the readout result, it was empirically determined as $0.15 \mathrm{px}$.

\section{Results}

\subsection{Perturbation Sensitivity of the Signal Tracking}

In this section the robustness of the image partitioning and the signal tracking is estimated. Perturbations, which are due to non-ideal cutting and aging of the groove, are simulated on synthetic data. To create a ground truth surface, a depth map is established by simulating the cutting effect. It is used as surface bump map during ray tracing using Povray [15] (right image of Fig. 5). The signal run found on the undistorted images is taken as ground truth signal.

To simulate aging, ellipses with random orientation are drawn in white and black over the resulting image. White ellipses model reflecting particles like dust whereas black model light-absorbing particles like rust. The ellipse length is $150 \mu \mathrm{m}$ at an aspect ratio of 20 and $1 / 3$ transparency. Realistic cutting artifacts are simulated as depth map bumps of $10 \mu \mathrm{m}$ diameter with normal-distributed heights. Their standard deviation is called roughness $\sigma_{\text {surf }}$. Such bumps usually originate from material inhomogeneities and non-ideal cutting. The influence of both perturbations on the SNR is shown in Fig. 6. To have an estimate about the strongest perturbation degrees, the processed images are also displayed.

It can be deduced that particles as well as roughness have influence on the accuracy of the signal reconstruction but the image partitioning does not fail even in images where no coarse structure is cognizable. The influence of particles on the surface is nearly linear for exponentially-rising particle count. Surface roughness has a stronger impact on the SNR as it disturbs the signal tracking more. However, in both cases highly-distorted surface images data could be used to extract the signal with an SNR satisfactory for further channel coding processing.

\subsection{Writing and Reading Data}

With the proposed methods from Section 2 and 3 , it is possible to track groove sections from one surface view. In the following, the steps necessary to 

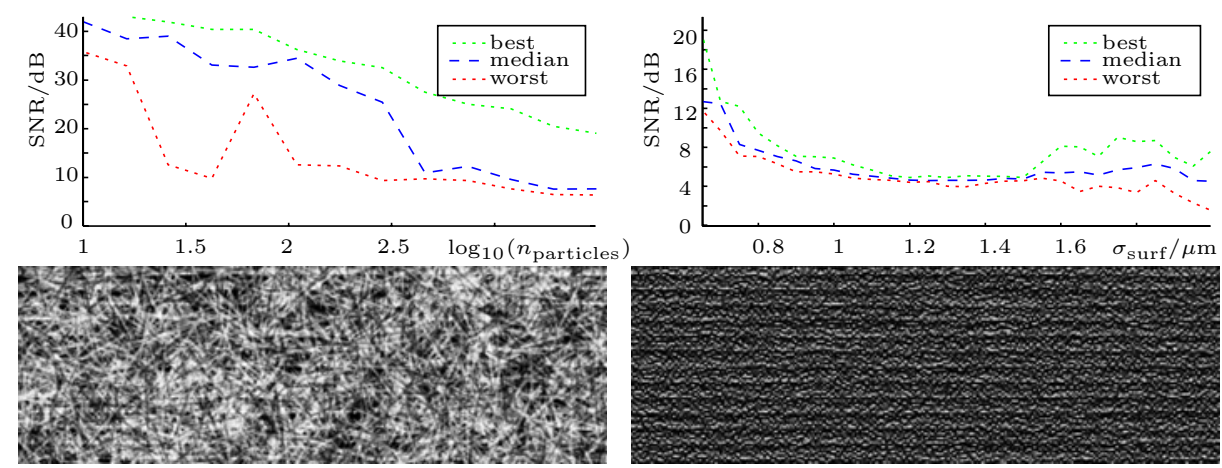

Fig. 6. Top: Signal to noise ratio for particle perturbations (left) and surface roughness (right). Bottom: Corresponding images for both perturbations with highest distortion tested. The views could be read out with $\mathrm{SNR}=7.7 \mathrm{~dB}$ and $\mathrm{SNR}=4.5 \mathrm{~dB}$.

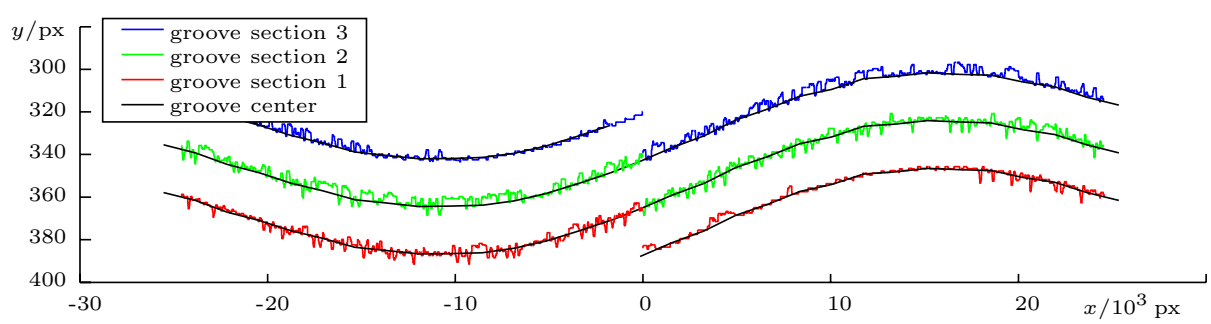

Fig. 7. The fusion of different groove segments at $x=0$. The large sinusoidal run of the groove center originates from the mechanical component wobbling during the readout.

reconstruct the whole groove run from several surface views are explained. The set-up to assemble the groove is as follows: The cylindrical mechanical component is mounted onto a turn table such that its axis of symmetry coincides with the rotation axis. By this, the component can be turned without changing its distance to the microscope. We assume that a rotation by a small angle $\theta$ results in an horizontal offset $\Delta$ in the microscope view. The ratio $r=\theta / \Delta$ is calibrated by estimating $\Delta$ using a minimization of the normalized cross correlation. Analog, vertical movement can be calibrated. Now image slices from different surface views can be stitched together to the surface view of one rotation.

After a whole surface view is created, the readout consisting of the partitioning and the signal tracking is performed. Next, the extracted groove sections are fused at the joint position where the right border of the whole surface view joins the left. At this position, groove section $n$ meets section $n+1$ (Fig. (7) such that the whole groove signal can be assembled. As last groove processing step, the signal is unbiased by subtracting the groove center from the signal. It is then demodulated re-using the sinusoidal parameter estimation derived in Section 2 . So the digital signal is sampled at positions $x_{n}=n \lambda_{s}-\phi_{s}$ with the estimates from Equations (5) and (10). To fulfill the Shannon sampling theorem [16], first 

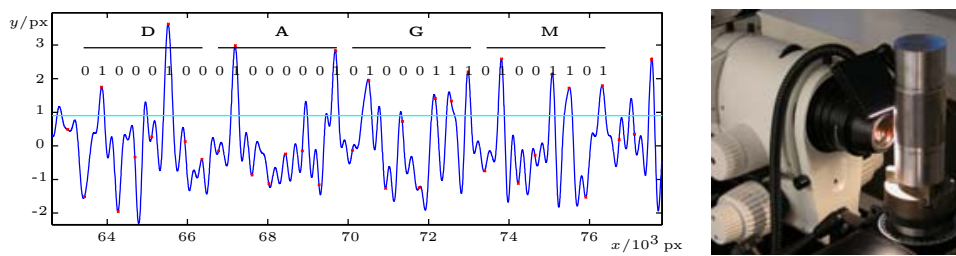

Fig. 8. Left: The extracted signal (blue) with sample positions (red), threshold (cyan) and the ASCII string "DAGM" read out. Right: Set-up for the readout.

the signal is low-pass filtered with wavelength $\lambda_{s} / 2$. A threshold is extracted automatically by a predefined initialization sequence.

To demonstrate writing and reading, we chose the ASCII string "DAGM" and modulated it using 2-ASK with every second bit set to 0 to ease phase estimation during the demodulation. The signal was written to a component of $49 \mathrm{~mm}$ diameter. Surface views were automatically stitched together to the overall surface image of size $48500 \mathrm{px} \times 768 \mathrm{px}$. The run of the groove center was determined using 100 estimates around the surface. All groove sections were tracked and combined to the reconstructed groove signal, which is shown in Fig. 8. The processing time for such a diameter is approximately $5 \mathrm{~min}$ for surface recording and $10 \mathrm{~min}$ for partitioning and tracking of 3 groove sections along one rotation. It can be seen all bits are demodulated correctly although the peak amplitude is only $\approx 3 \mathrm{px}$ which is hardly cognizable in the surface views. The strongest perturbation is a nearly-periodic signal component with wavelength $2 / 3 \lambda_{s}$ which is due to resonance during the cutting process. Investigations on various surfaces with different data showed that the readout results have high repeatability and are in combination with a channel code very robust.

\section{$5 \quad$ Summary and Conclusion}

An optical readout procedure for data mechanically stored as a groove varying by only $3 \mu \mathrm{m}$ in depth has been presented. The groove which is wound around a cylindrical mechanical part is reconstructed using directed illumination. The two key problems which were solved are the image partitioning of the periodicallyoccurring groove sections and the tracking of the groove border. The image partitioning into single groove sections is based on the vertical spectral analysis of the image texture. For precise and robust PSD estimates, the average periodogram method was combined with an ML estimation. Analog, a phase estimation algorithm was derived which combines the estimations of multiple realizations. For this purpose, an adapted phase unwrapping is used. As solution of the second key problem, a Hidden Markov Model for the tracking of the groove border was derived which consists of one node per pixel. Its observation probabilities model the probability of the groove border passing the specific node whereas its transition probabilities model the properties of the Piezo tool which has cut the groove. We demonstrated the robustness of the image partitioning and the signal 
tracking by perturbing synthetic ground truth data rendered by Povray with realistic distortions. We further demonstrated reading out data from real surfaces. Our fully automatic system is running reliable as on-line demo and is frequently presented at different events in our lab. We further research in transferring this readout method to arbitrary-curved surfaces which are created during a milling process. It is planned to modulate the depth of the milling head by the analog signal which corresponds to the variation of the Piezo tool excitation here.

\section{References}

1. Denkena, B., Ostermann, J., Becker, C., Spille, C.: Mechanical Information Storage by Use of an Excited Turning Tool. Annals of the German Academic Society for Production Engineering 1(1), 25-30 (2007)

2. Isailović, J.: 2.4: Capacitive Videodiscs. In: Videodisc and Optical Memory Systems. Prentice-Hall, Englewood Cliffs (1985)

3. Takita, A., Yamamoto, H., Hayasaki, Y., Nishida, N.: Three-Dimensional Optical Storage Inside Transparent Materials. Optics Letters 21(24), 2023-2030 (1996)

4. Schwartz, M.: 1: Introduction to Information Transmission. In: Information Transmission, Modulation, and Noise. McGraw-Hill, New York (1970)

5. Grossmann, P.: Depth from focus. Pattern Recognition Letters 5(1), 63-69 (1987)

6. Namboodiri, V.P., Chaudhuri, S.: On defocus, diffusion and depth estimation. Pattern Recognition Letters 28(3), 311-319 (2007)

7. Falkenhagen, L.: Depth Estimation from Stereoscopic Image Pairs Assuming Piecewise Continuos Surfaces. In: Proc. European Workshop on Combined Real and Synthetic Image Processing for Broadcast and Video Productions, Hamburg, Germany (November 1994)

8. Horn, B.K.P., Brooks, M.J. (eds.): Shape From Shading. MIT Press, Cambridge (1989)

9. Tian, B., Barron, J.L.: Reproduction of sound signal from gramophone records using 3d scene reconstruction. In: Irish Machine Vision and Image Processing Conference (2006)

10. Sanderson, A.C., Weiss, L.E., Nayar, S.K.: Structured Highlight Inspection of Specular Surfaces. IEEE Transactions on Pattern Analysis and Machine Intelligence 10(1), 44-55 (1988)

11. Schaper, D.: Automated Quality Control for Micro-Technology Components Using a Depth From Focus Approach. In: Fifth IEEE Southwest Symposium on Image Analysis and Interpretation, Sante Fe, USA, April 2002, pp. 50-54 (2002)

12. Kay, S.M.: Modern Spectral Estimation. Prentice-Hall, Englewood Cliffs (1988)

13. Harris, F.J.: On the Use of Windows for Harmonic Analysis with the Discrete Fourier Transform. Proceedings of the IEEE 66(1), 51-83 (1978)

14. Viterbi, A.J.: Error Bounds for Convolutional Codes and an Asymptotically Optimum Decoding algorithm. IEEE Transactions on Information Theory 13(2), 260269 (1967)

15. Povray: The persistence of vision raytracer, http://www.povray.org/

16. Shannon, C.E.: Communication in the Presence of Noise. Proceedings of the IRE 37(1), 10-21 (1949) 